\title{
Distribuição Volumétrica de Calda Produzidas pelas Pontas PUlVerizaÇÃo XR, TP E TJ SOB Diferentes Condições OPERACIONAIS ${ }^{1}$
}

\author{
Volumetric Distribution of the Spray Nozzle Tips XR, TP and TJ under Different Operational \\ Conditions
}

BAUER, F.C. ${ }^{2}$ e RAETANO, C.G. ${ }^{3}$

\begin{abstract}
RESUMO - Foram avaliados os perfis de distribuição volumétrica das pontas de pulverização de jato plano "Extended Range" XR 8004, "Teejet" TP 8004 e "TwinJet" TJ60-8004, em mesa de teste construída segundo a norma ISO 5.682-1:1996, sendo as pontas de pulverização posicionadas a 30, 40 e $50 \mathrm{~cm}$ de altura da mesa coletora, submetidas à pressão de 200 e $300 \mathrm{kPa}$, e posicionadas com ângulos de 30 e $45^{\circ}$ nos sentidos horário e anti-horário e na posição vertical $\left(0^{\circ}\right)$. As pontas TJ60-8004 foram analisadas nas mesmas alturas, porém somente na posição vertical. Avaliaram-se 10 unidades de cada tipo de ponta, instaladas isoladamente na mesa. A distribuição média do volume pulverizado, coletado nas provetas, foi inserida em programa computacional, que permitiu a simulação do perfil de distribuição de uma barra pulverizadora com 24 bicos, em espaçamentos variáveis, obtendo-se ao final o coeficiente de variação, o desvio-padrão e a média do volume. Os resultados mostraram que a uniformidade de distribuição é menos influenciada pela variação da altura da barra quando o espaçamento entre bicos é reduzido e que há necessidade de adequação do espaçamento quando se utiliza ângulo na barra pulverizadora.
\end{abstract}

Palavras-chave: ângulo de aplicação, bicos, pulverizador de barra, tecnologia de aplicação, coeficiente de variação.

ABSTRACT - The deposition pattern of flat fan nozzles "Extended Range" XR 8004, "Teejet" TP 8004 and "TwinJet" TJ60-8004 were evaluated, using a patternator table, built in accordance with ISO 5.682-1:1996, with the nozzle typs placed at 30, 40 and $50 \mathrm{~cm}$ above the patternator table being submitted to pressures of 200 and $300 \mathrm{kPa}$ and angles of $30^{\circ}$ and $45^{\circ}$, clockwise and counterclockwise and in the vertical position $\left(0^{\circ}\right)$. The TJ60-8004 nozzle units were installed at the same heights, but only in the vertical position. The mean collected spray volume distribution of 10 units of each type, in each configuration, was entered in a computer program, which simulated the deposition volume pattern of a boom sprayer with 24 nozzles placed in different spacings with, the coefficient of variation, standard deviation and mean volume being obtained from each simulation. The results showed that the distribution uniformity is less vulnerable to changes in height as the nozzle spacing is reduced and that adjustment on nozzle spacing is needed if any angle is used.

Key words: coefficient of variation, spray angle, nozzles, boom sprayer, spray deposition.

\section{INTRODUÇÃO}

O modelo de agricultura adotado nos diversos sistemas de produção - notadamente para as culturas que ocupam extensas áreas, como algodão, soja, milho, entre outras - é dependente do uso de produtos fitossanitários, que atuam como importante componente no manejo de culturas.

A uniformidade na distribuição da calda aplicada, em pulverizações de produtos fitossanitários, é dada pelas condições de montagem e de operação do equipamento, como

Recebido para publicação em 29.8.2003 e na forma revisada em 18.6.2004.

2 Eng.-Agr., D.S., Bolsista CNPq, Pesquisador Dep. de Produção Vegetal, Defesa Fitossanitária, Faculdade de Ciências Agronômicas, Universidade Estadual Paulista - UNESP, Caixa Postal 237, 18603-970 Botucatu-SP, <fcbauer@ mail.uniderp.br>; ${ }^{3}$ Eng.-Agr., Prof. Assist., D.S., Dep. de Produção Vegetal - UNESP. 
espaçamento entre bicos, altura da barra, ângulo de abertura dos bicos e pressão de trabalho (Perecin et al., 1994). A ponta, órgão responsável pela emissão das gotas e, por isso, considerado o componente mais importante do equipamento pulverizador, é que determina diversos fatores relacionados à qualidade da aplicação, entre eles a vazão e a uniformidade de distribuição do líquido, os quais devem apresentar a menor variação possível ao longo da barra.

Downer et al. (1997) afirmam que o objetivo de muitos pesquisadores é reduzir a quantidade de produto aplicado. Com doses e volumes reduzidos, a uniformidade na distribuição deve ser alcançada; caso contrário, a dose que chega ao alvo poderá ser insuficiente para causar o efeito biológico desejável, ou a dose poderá ser excessiva, determinando desperdício de produto, perdas e contaminação do ambiente.

Há atualmente no mercado pontas desenvolvidas para as mais diversas aplicações e situações. Segundo Galli et al. (1983) e Matthews (2000), cada uma delas possui um padrão de distribuição característico, que determina a altura da ponta em relação ao alvo e o espaçamento entre elas na barra pulverizadora.

A partir do padrão de distribuição de bicos individuais, pode-se simular a sobreposição de vários bicos, tal qual ocorre na barra pulverizadora, calculando-se, então, o coeficiente de variação (CV) da sobreposição resultante; segundo Christofoletti (1997), quanto menor esse valor, menos desuniforme será a distribuição.

Os padrões de deposição individual dos bicos são determinados em bancadas ou mesas de teste, onde canaletas realizam a coleta do líquido a distâncias predeterminadas e o depositam em recipientes individuais (Chapple et al., 1993). No Brasil, é freqüente o uso de barras pulverizadoras dotadas de bicos espaçados de 0,40 ou $0,50 \mathrm{~m}$ entre si; no entanto, dependendo do tipo de ponta de pulverização, da altura da barra e da pressão de trabalho, esses espaçamentos não são adequados à melhor distribuição do líquido.

Assim, este trabalho teve como objetivo determinar, em laboratório, quais espaçamentos e altura de barra produzem melhor distribuição da pulverização, nas pressões selecionadas, com as pontas XR, TP e TJ608004 posicionadas em diferentes ângulos de aplicação.

\section{MATERIAL E MÉTODOS}

O trabalho foi desenvolvido no Laboratório de Tecnologia de Aplicação de Defensivos Agrícolas do Departamento de Produção Vegetal - Defesa Fitossanitária da Faculdade de Ciências Agronômicas, UNESP-Botucatu, SP.

A avaliação das condições operacionais, visando melhor uniformidade de distribuição da pulverização, foi feita em mesa de teste para pontas de pulverização hidráulica construída conforme a norma ISO 5.682-1:1996, descrita detalhadamente por Bauer (2002).

Foram utilizadas 10 unidades de cada tipo das pontas de jato plano "Extended Range" XR 8004, "TeeJet" TP 8004 e "TwinJet" TJ608004, fabricadas pela Spraying System Co. Cada uma das 10 unidades dos diferentes tipos de ponta foi instalada, isoladamente, nas alturas de 0,30, 0,40 e 0,50 m, em relação à superfície da mesa. Em cada altura as pontas foram submetidas às pressões de 200 e $300 \mathrm{kPa}$ e colocadas na posição vertical $\left(0^{\circ}\right)$ e nos ângulos de 30 e $45^{\circ}$, nos sentidos horário e anti-horário. As pontas de jato plano duplo TJ60-8004 foram colocadas somente na posição vertical. Portanto, foram avaliadas 10 unidades de dois tipos de pontas em 30 configurações diferentes (três alturas, duas pressões e cinco ângulos) e 10 unidades da terceira ponta em seis configurações (três alturas e duas pressões).

Após a instalação de cada ponta no centro da barra, colocou-se o sistema hidráulico em funcionamento até que o fluxo de líquido emitido se estabilizasse, quando, então, se iniciava a coleta do líquido pulverizado por um período de 60 segundos, momento em que se encerrava a coleta, procedendo-se à leitura dos volumes de cada proveta. Dessa forma, a amostragem foi feita em 10 unidades de cada tipo de ponta, sendo a pulverização feita 10 vezes em cada configuração.

Encerradas as pulverizações, foi calculada a deposição média obtida das 10 unidades de 
cada tipo de ponta em estudo em cada uma das condições operacionais, possibilitando o cálculo do padrão médio da distribuição volumétrica. A partir desses valores, iniciouse o estudo de sobreposição, individualmente, para cada uma das configurações. Para isso, foi desenvolvido um programa computacional, descrito por Bauer (2002), similar ao utilizado por Perecin et al. (1994), no qual, a partir dos valores de deposição das pontas individuais, é simulada a deposição da pulverização em toda a barra.

O programa funciona de forma independente da configuração e considera o número de provetas em que houve deposição de líquido durante as pulverizações, bem como o número de provetas que serão sobrepostas (sabendose que o líquido contido em cada proveta corresponde ao volume proveniente de cada uma canaleta coletora de $5 \mathrm{~cm}$ de largura), possibilitando a simulação de uma barra hipotética, em que se pode trabalhar com um número infinito de bicos e com sobreposições variáveis de 5 em $5 \mathrm{~cm}$, até o limite da faixa de deposição da ponta. Esse programa fornece, ao final das análises, o coeficiente de variação $(\mathrm{CV})$ do resultado da sobreposição, o desvio-padrão e a média do volume aplicado nas provetas.

A simulação do uso das pontas em barra foi feita considerando as constatações de Perecin et al. (1998), que indicam ser esse procedimento aceitável do ponto de vista estatístico, uma vez que a causa das variabilidades sistemática (CVS) e aleatória (CVA) são dependentes de causas diferentes. Por isso, neste trabalho, serão apresentados valores relativos ao CV resultante (CVG) dos dois anteriores, calculado pela equação 1 , por eles proposta.

$$
\mathrm{CVG}=\left[(\mathrm{CVA})^{2}+(\mathrm{CVS})^{2}\right]^{1 / 2}\left(\mathrm{E}_{1}\right)
$$

\section{RESULTADOS E DISCUSSÃO}

O programa computacional, aliado à mesa de teste de distribuição volumétrica, permite a obtenção de número de combinações até o limite da faixa de deposição da ponta utilizada, com variações de espaçamento a cada $5 \mathrm{~cm}$, mostrando ser importante ferramenta no estudo de pontas de pulverização hidráulica em laboratório, além de apontar a necessidade de adequação dos espaçamentos entre bicos comumente presentes nas barras para melhor distribuição da pulverização.

Os coeficientes de variação, calculados a partir das vazões médias, se mantiveram sempre abaixo de 3,9\%, indicando bom nivel de repetibilidade e equivalência quanto à vazão e distribuição do volume aplicado.

Os padrões de deposição média em cada tipo de ponta são apresentados nas Figuras 1 a 3, respectivamente para as pontas XR 8004, TP 8004 e TJ60-8004, onde são mostradas na abscissa as canaletas da mesa de teste com $5 \mathrm{~cm}$ de largura e, na ordenada, as porcentagens dos volumes depositados.

Galli et al. (1983) observaram, em alguns casos, variações consideráveis na vazão, em relação àquelas indicadas pelos fabricantes, fato que os levou a recomendar estudo mais detalhado das pontas antes de utilizá-las. Da mesma forma, Perecin et al. (1998) consideraram esse procedimento recomendável, em razão das diferenças encontradas entre os padrões de distribuição volumétrica de diferentes unidades de uma mesma ponta. Contudo, no presente trabalho, não se constatou esse tipo de diferença; a vazão, nas unidades utilizadas, variou de 1,28 a $1,34 \mathrm{~L} \mathrm{~min}^{-1}$ à pressão de $200 \mathrm{kPa}$ e de 1,54 a $1,59 \mathrm{~L} \mathrm{~min}^{-1}$ com $300 \mathrm{kPa}$, lembrando que, segundo o fabricante, a vazão para essas pontas, nas pressões utilizadas, é de 1,29 e $1,58 \mathrm{~L} \mathrm{~min}^{-1}$. Isso se deve à grande evolução em se tratando de material e processo de fabricação das pontas, fazendo com que as diferenças entre as pontas de um mesmo tipo sejam muito pequenas e permaneçam dentro dos limites de tolerância. Cabe ressaltar que as normas vigentes para avaliação da vazão de pontas de pulverização hidráulica são específicas para essa finalidade e não foram utilizadas neste trabalho, pois o objetivo principal foi a avaliação do padrão de deposição e não da vazão.

Os perfis de distribuição volumétrica das pontas submetidas às diferentes alturas e pressões utilizadas demonstram haver grande influência na largura da distribuição quando os bicos são posicionados com algum ângulo em relação à vertical. O uso das pontas com $45^{\circ}$ de inclinação, no sentido horário, resultou em aumento na largura da deposição superior ao obtido com essa mesma ponta na posição 
vertical, porém com a barra $10 \mathrm{~cm}$ mais alta (Figuras 1 e 2). Isso mostra que a angulação dos bicos na barra tem efeito parecido quando se relaciona à distribuição da pulverização; a

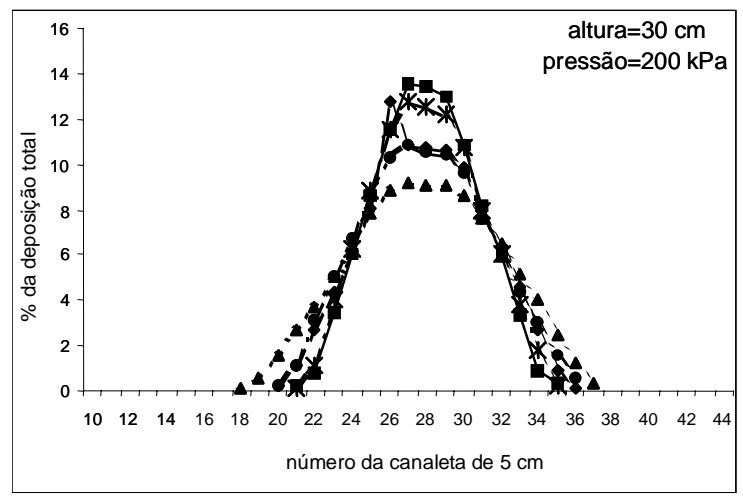

(A)

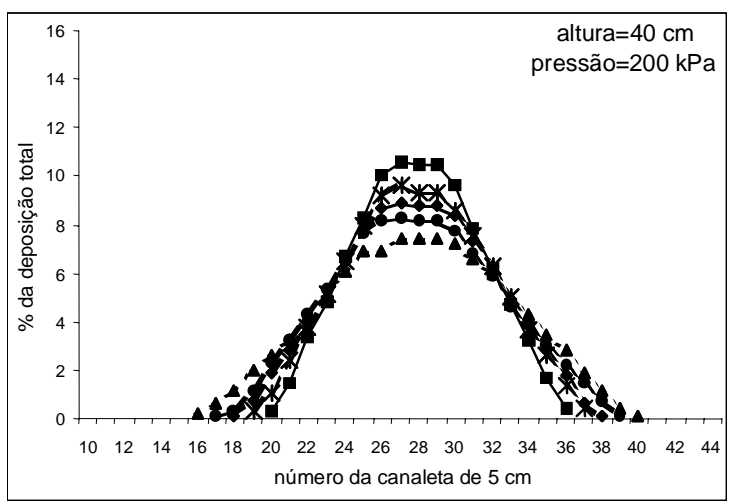

(C)

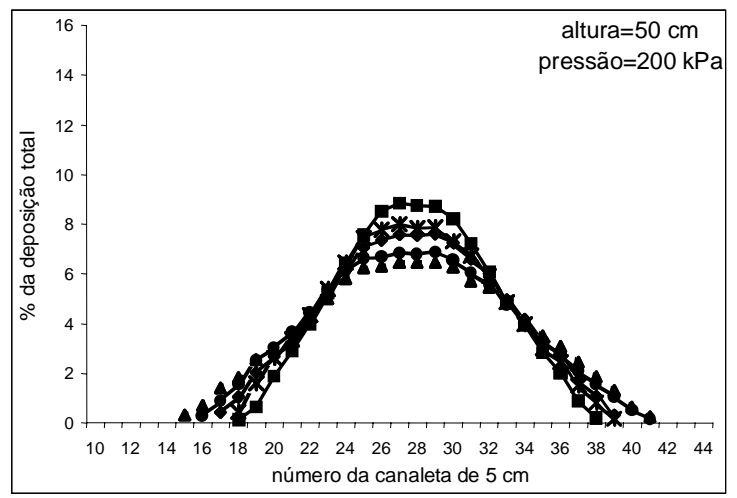

(E) elevação da barra reflete negativamente na sobreposição, indicando a necessidade de adequação do espaçamento entre bicos quando posicionados em ângulo na barra pulverizadora.

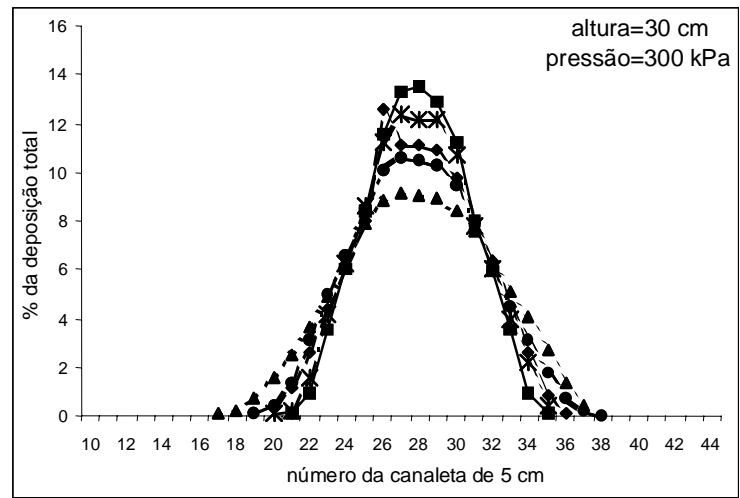

(B)

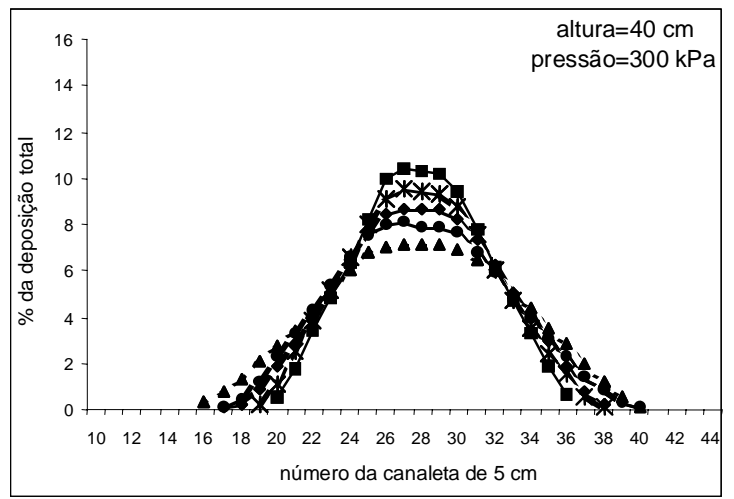

(D)

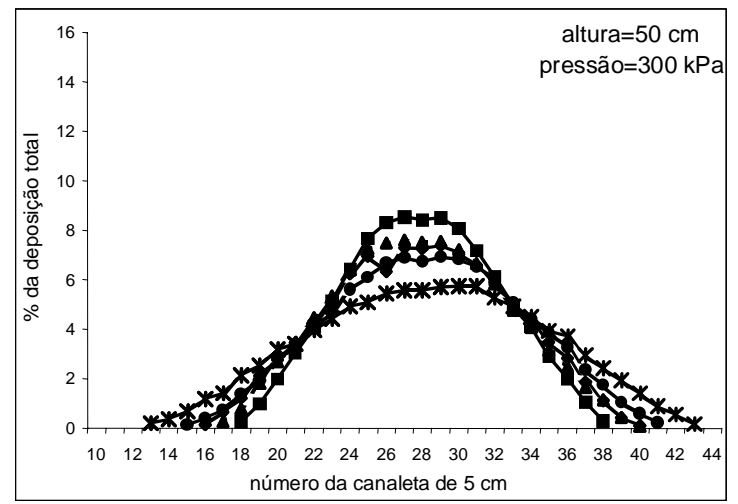

$(\mathrm{F})$

\section{$\longrightarrow$ Vertical $\quad \longrightarrow-30^{\circ}$ horário $\quad-\cdots-45^{\circ}$ horário $\quad-*-30^{\circ}$ anti-horário $\quad-\bullet-45^{\circ}$ anti-horário}

Figura 1 - Perfis de distribuição volumétrica de pontas XR 8004 submetidas a 200 (A, C e E) e 300 kPa (B, D e F) e a 30 (A e B), 40 (C e D) e $50 \mathrm{~cm}$ de altura (E e F), em cinco ângulos de pulverização, com distribuição do volume (\%) em cada canaleta de $5 \mathrm{~cm}$, em mesa de teste para pontas de pulverização hidráulica. 


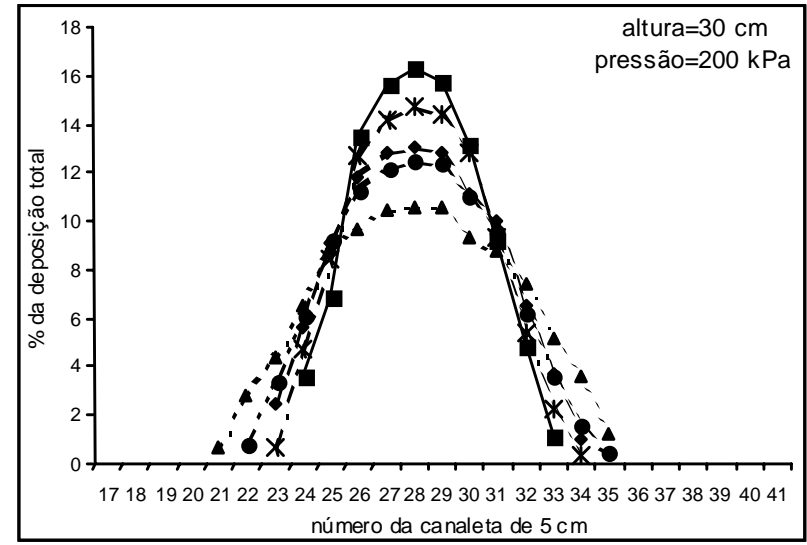

(A)

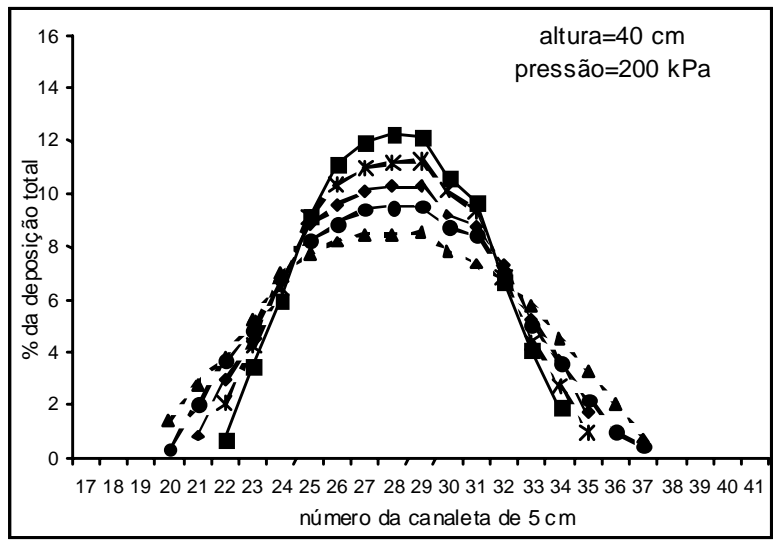

(C)

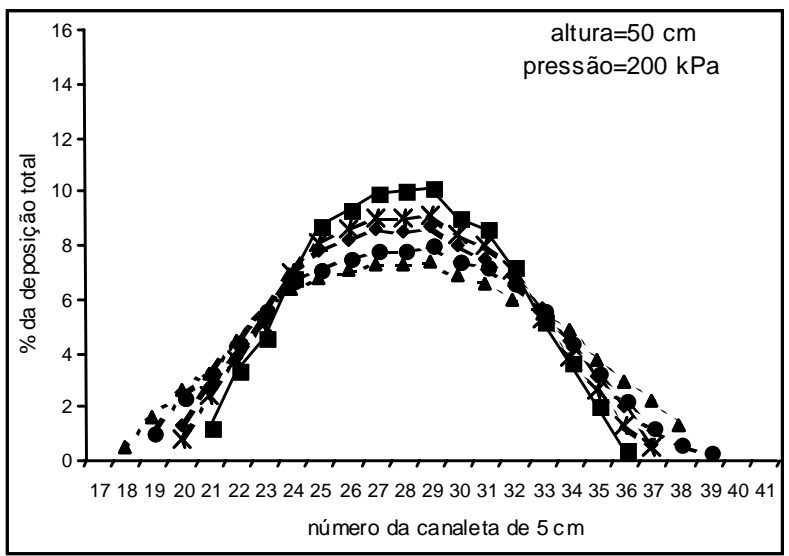

(E)

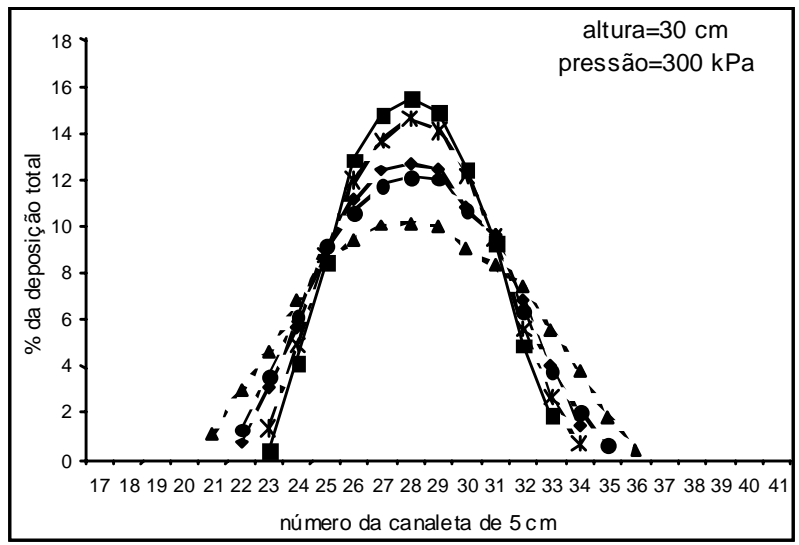

(B)

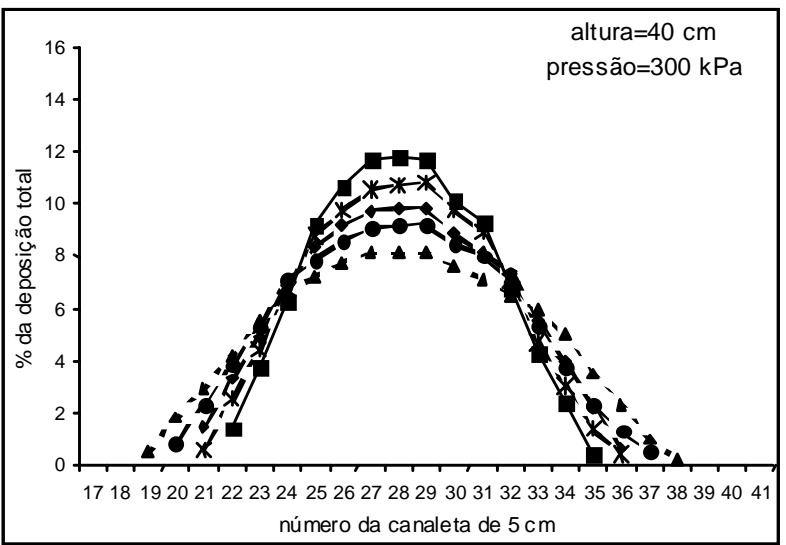

(D)

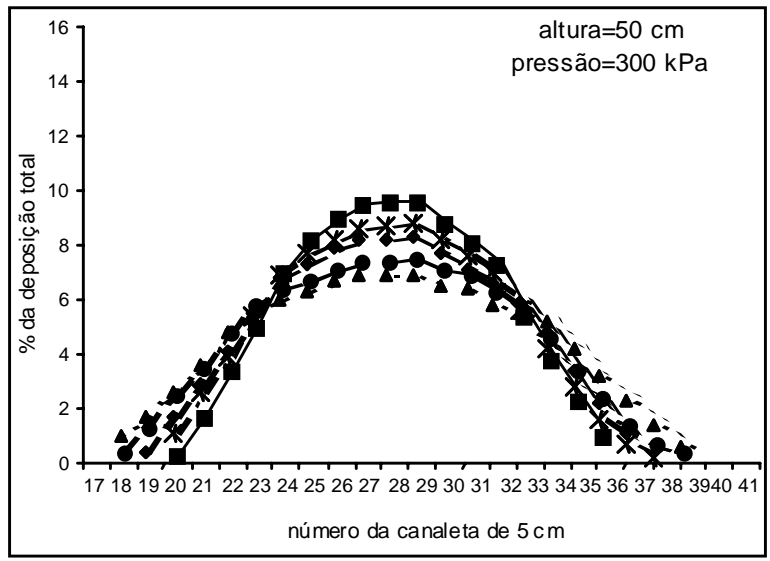

(F)

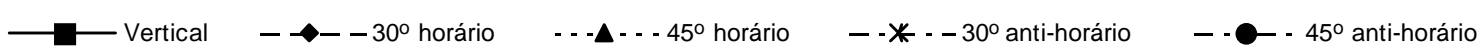

Figura 2 - Perfis de distribuição volumétrica de pontas TP 8004 submetidas a 200 (A, C e E) e 300 kPa (B, D e F) e a 30 (A e B), 40 (C e D) e $50 \mathrm{~cm}$ de altura (E e F), em cinco ângulos de pulverização, com distribuição do volume (\%) em cada canaleta de $5 \mathrm{~cm}$, em mesa de teste para pontas de pulverização hidráulica. 

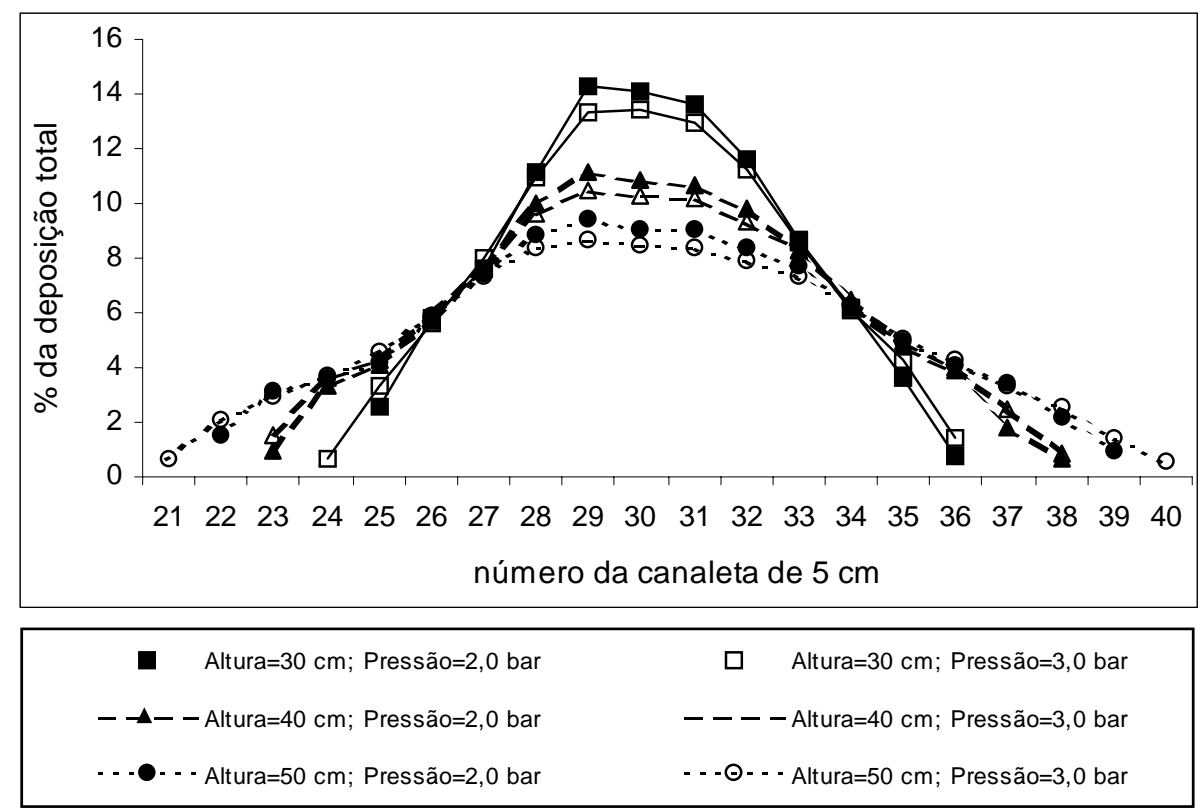

Figura 3 - Perfis de distribuição volumétrica de pontas TJ60-8004 na posição vertical submetidas a 200 e $300 \mathrm{kPa}$ e a 30 , 40 e $50 \mathrm{~cm}$ de altura, com distribuição do volume depositado (\%) em cada canaleta de $5 \mathrm{~cm}$, em mesa de teste para pontas de pulverização hidráulica.

O aumento da pressão, de 200 para $300 \mathrm{kPa}$, não teve efeito significativo na distribuição da pulverização, tendo sido verificado que, em algumas configurações, houve aumento da faixa de deposição de até $10 \%$. Todavia, o volume depositado nessa faixa excedente não foi superior a $1 \%$ da deposição total. É oportuno acrescentar que esse efeito só foi observado quando a barra foi posicionada nas alturas de 40 e $50 \mathrm{~cm}$, sendo mais pronunciado quando estavam em ângulo na barra, fato que indica haver certa instabilidade na largura da deposição quando se utiliza algum ângulo diferente da posição vertical.

A angulação dos bicos na barra pulverizadora teve efeito direto na faixa de deposição, alterando o espalhamento, o que se justifica pelo aumento da distância do bico até o alvo.

Assim, como observado por Perecin et al. (1998), o efeito da altura da barra pulverizadora em relação ao alvo foi similar para todas as pontas e em todos os ângulos estudados, ocorrendo somente aumento na faixa de deposição.

Embora os resultados permitam estudo de outros fatores, do ponto de vista prático, o coeficiente de variação (CV) sob a barra é o indicador mais importante relacionado à homogeneidade da deposição. Por isso, neste trabalho, optou-se por avaliar somente o CV, em função do grande número de configurações (36) e da grande diferença entre elas. As configurações e seus resultados, indicando a largura total da deposição, o maior espaçamento entre bicos cuja simulação resultou em CV imediatamente inferior a 7\%, bem como a porcentagem da sobreposição em simulação de barra com 24 bicos, podem ser observados nos Quadros 1 e 2. Optou-se por selecionar as configurações com $\mathrm{CV}$ abaixo de $7 \%$, por ser esse valor adotado em vários países da Europa, entre eles a Itália e a Suécia.

Ao observar os Quadros 1 e 2, nota-se que, qualquer que seja a configuração, sempre houve a necessidade de aproximar os bicos para conseguir os níveis de CV desejados; esses níveis sempre estiveram acima da recomendação de 30\% de sobreposição, indicando a necessidade de adequação do espaçamento entre bicos na barra pulverizadora em relação ao ângulo de aplicação. Essa observação já havia sido feita por Perecin et al. (1994), que alertavam sobre a conveniência de utilizar espaçamentos entre bicos menores que $50 \mathrm{~cm}$. 
Quadro 1 - Largura da deposição (cm), espaçamento entre bicos (cm) e sobreposição (\%) obtidos da simulação de pulverização com CV abaixo de 7\%, em pontas XR 8004 e TP 8004, indicando pressão, altura da barra e ângulo do bico

\begin{tabular}{|c|c|c|c|c|c|c|}
\hline Ponta & $\begin{array}{c}\text { Altura } \\
(\mathrm{cm})\end{array}$ & $\begin{array}{c}\text { Pressão } \\
(\mathrm{kPa})\end{array}$ & Ângulo/sentido & $\begin{array}{c}\text { Deposição total } \\
(\mathrm{cm})\end{array}$ & $\begin{array}{c}\text { Espaçamento com } \\
\mathrm{CV}<7 \%(\mathrm{~cm})\end{array}$ & $\begin{array}{c}\text { Sobreposição } \\
(\%)\end{array}$ \\
\hline \multirow{6}{*}{ XR 8004} & \multirow{2}{*}{30} & 200 & $\begin{array}{l}\text { vertical } \\
30^{\circ} \text { horário } \\
30^{\circ} \text { anti-horário } \\
45^{\circ} \text { horário } \\
45^{\circ} \text { anti-horário }\end{array}$ & $\begin{array}{l}65 \\
75 \\
65 \\
90 \\
80 \\
\end{array}$ & $\begin{array}{l}35 \\
30 \\
40 \\
55 \\
50 \\
\end{array}$ & $\begin{array}{l}46 \\
47 \\
38 \\
39 \\
37 \\
\end{array}$ \\
\hline & & 300 & $\begin{array}{l}\text { vertical } \\
30^{\circ} \text { horário } \\
30^{\circ} \text { anti-horário } \\
45^{\circ} \text { horário } \\
45^{\circ} \text { anti-horário }\end{array}$ & $\begin{array}{l}65 \\
75 \\
70 \\
95 \\
85 \\
\end{array}$ & $\begin{array}{l}40 \\
25 \\
40 \\
60 \\
50 \\
\end{array}$ & $\begin{array}{l}38 \\
67 \\
43 \\
37 \\
44 \\
\end{array}$ \\
\hline & \multirow{2}{*}{40} & 200 & $\begin{array}{l}\text { vertical } \\
30^{\circ} \text { horário } \\
30^{\circ} \text { anti-horário } \\
45^{\circ} \text { horário } \\
45^{\circ} \text { anti-horário }\end{array}$ & $\begin{array}{c}75 \\
85 \\
85 \\
105 \\
100 \\
\end{array}$ & $\begin{array}{l}50 \\
60 \\
55 \\
70 \\
65 \\
\end{array}$ & $\begin{array}{l}33 \\
29 \\
35 \\
33 \\
35 \\
\end{array}$ \\
\hline & & 300 & $\begin{array}{l}\text { vertical } \\
30^{\circ} \text { horário } \\
30^{\circ} \text { anti-horário } \\
45^{\circ} \text { horário } \\
45^{\circ} \text { anti-horário }\end{array}$ & $\begin{array}{c}85 \\
95 \\
85 \\
115 \\
100 \\
\end{array}$ & $\begin{array}{l}50 \\
60 \\
55 \\
75 \\
65 \\
\end{array}$ & $\begin{array}{l}41 \\
37 \\
35 \\
35 \\
35 \\
\end{array}$ \\
\hline & \multirow{2}{*}{50} & 200 & $\begin{array}{l}\text { vertical } \\
30^{\circ} \text { horário } \\
30^{\circ} \text { anti-horário } \\
45^{\circ} \text { horário } \\
45^{\circ} \text { anti-horário }\end{array}$ & $\begin{array}{c}95 \\
105 \\
100 \\
125 \\
120 \\
\end{array}$ & $\begin{array}{l}60 \\
70 \\
65 \\
85 \\
70 \\
\end{array}$ & $\begin{array}{l}37 \\
33 \\
35 \\
32 \\
42 \\
\end{array}$ \\
\hline & & 300 & $\begin{array}{l}\text { vertical } \\
30^{\circ} \text { horário } \\
30^{\circ} \text { anti-horário } \\
45^{\circ} \text { horário } \\
45^{\circ} \text { anti-horário }\end{array}$ & $\begin{array}{c}95 \\
115 \\
110 \\
140 \\
125 \\
\end{array}$ & $\begin{array}{l}60 \\
75 \\
75 \\
70 \\
75 \\
\end{array}$ & $\begin{array}{l}37 \\
35 \\
32 \\
50 \\
40 \\
\end{array}$ \\
\hline \multirow{6}{*}{ TP 8004} & \multirow{2}{*}{30} & 200 & $\begin{array}{l}\text { vertical } \\
30^{\circ} \text { horário } \\
30^{\circ} \text { anti-horário } \\
45^{\circ} \text { horário } \\
45^{\circ} \text { anti-horário }\end{array}$ & $\begin{array}{l}55 \\
65 \\
60 \\
70 \\
70 \\
\end{array}$ & $\begin{array}{l}20 \\
25 \\
35 \\
30 \\
40\end{array}$ & $\begin{array}{l}64 \\
62 \\
62 \\
57 \\
43\end{array}$ \\
\hline & & 300 & $\begin{array}{l}\text { vertical } \\
30^{\circ} \text { horário } \\
30^{\circ} \text { anti-horário } \\
45^{\circ} \text { horário } \\
45^{\circ} \text { anti-horário }\end{array}$ & $\begin{array}{l}60 \\
70 \\
60 \\
85 \\
75 \\
\end{array}$ & $\begin{array}{l}35 \\
40 \\
35 \\
50 \\
45 \\
\end{array}$ & $\begin{array}{l}42 \\
43 \\
42 \\
41 \\
40\end{array}$ \\
\hline & \multirow{2}{*}{40} & 200 & $\begin{array}{l}\text { vertical } \\
30^{\circ} \text { horário } \\
30^{\circ} \text { anti-horário } \\
45^{\circ} \text { horário } \\
45^{\circ} \text { anti-horário }\end{array}$ & $\begin{array}{l}70 \\
80 \\
80 \\
95 \\
95 \\
\end{array}$ & $\begin{array}{l}40 \\
50 \\
45 \\
- \\
- \\
\end{array}$ & $\begin{array}{c}43 \\
37 \\
44 \\
- \\
- \\
\end{array}$ \\
\hline & & 300 & $\begin{array}{l}\text { vertical } \\
30^{\circ} \text { horário } \\
30^{\circ} \text { anti-horário } \\
45^{\circ} \text { horário } \\
45^{\circ} \text { anti-horário }\end{array}$ & $\begin{array}{c}70 \\
90 \\
80 \\
100 \\
100 \\
\end{array}$ & $\begin{array}{l}45 \\
55 \\
50 \\
65 \\
60 \\
\end{array}$ & $\begin{array}{l}36 \\
39 \\
37 \\
35 \\
40 \\
\end{array}$ \\
\hline & \multirow{2}{*}{50} & 200 & $\begin{array}{l}\text { vertical } \\
30^{\circ} \text { horário } \\
30^{\circ} \text { anti-horário } \\
45^{\circ} \text { horário } \\
45^{\circ} \text { anti-horário }\end{array}$ & $\begin{array}{c}80 \\
95 \\
95 \\
110 \\
110 \\
\end{array}$ & $\begin{array}{l}50 \\
60 \\
60 \\
75 \\
70 \\
\end{array}$ & $\begin{array}{l}37 \\
37 \\
37 \\
32 \\
36 \\
\end{array}$ \\
\hline & & 300 & $\begin{array}{l}\text { vertical } \\
30^{\circ} \text { horário } \\
30^{\circ} \text { anti-horário } \\
45^{\circ} \text { horário } \\
45^{\circ} \text { anti-horário }\end{array}$ & $\begin{array}{c}90 \\
100 \\
95 \\
115 \\
120 \\
\end{array}$ & $\begin{array}{l}55 \\
65 \\
60 \\
80 \\
70 \\
\end{array}$ & $\begin{array}{l}39 \\
35 \\
37 \\
30 \\
42 \\
\end{array}$ \\
\hline
\end{tabular}


Quadro 2 - Largura da deposição (cm), espaçamento entre bicos e sobreposição (\%) obtidos da simulação de pulverização com CV abaixo de 7\%, em pontas TJ60-8004, indicando pressão, altura da barra e ângulo do bico

\begin{tabular}{|c|c|c|c|c|c|}
\hline $\begin{array}{c}\text { Altura } \\
(\mathrm{cm})\end{array}$ & $\begin{array}{c}\text { Pressão } \\
(\mathrm{kPa})\end{array}$ & Ângulo/sentido & $\begin{array}{c}\text { Deposição total } \\
(\mathrm{cm})\end{array}$ & $\begin{array}{c}\text { Espaçamento com } \\
\mathrm{CV}<7 \%(\mathrm{~cm})\end{array}$ & $\begin{array}{c}\text { Sobreposição } \\
(\%)\end{array}$ \\
\hline \multirow{2}{*}{30} & 200 & vertical & 60 & 35 \\
& 300 & vertical & 65 & 40 \\
\hline \multirow{2}{*}{40} & 200 & vertical & 80 & 45 \\
& 300 & vertical & 85 & 50 \\
\hline \multirow{2}{*}{50} & 200 & vertical & 90 & 55 \\
& 300 & vertical & 100 & 60 \\
\hline
\end{tabular}

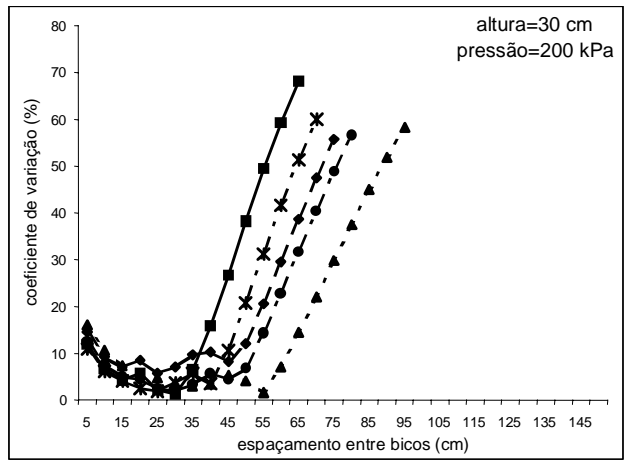

(A)

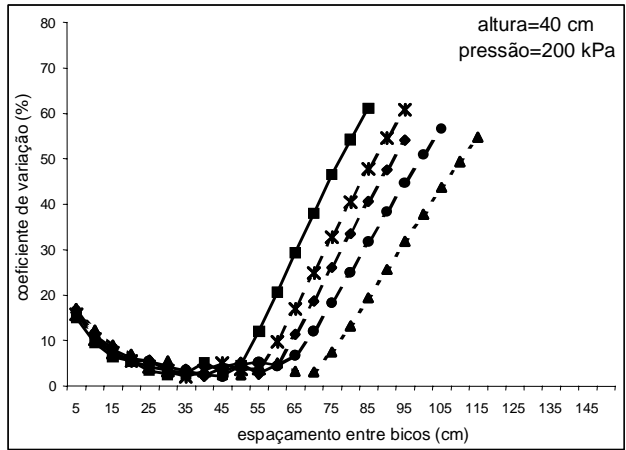

(C)

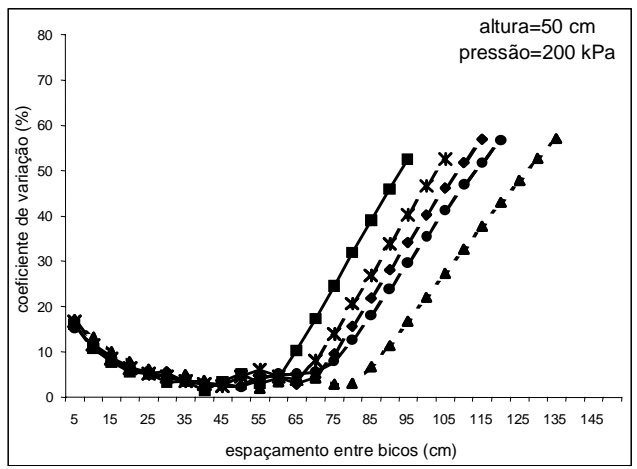

(D)

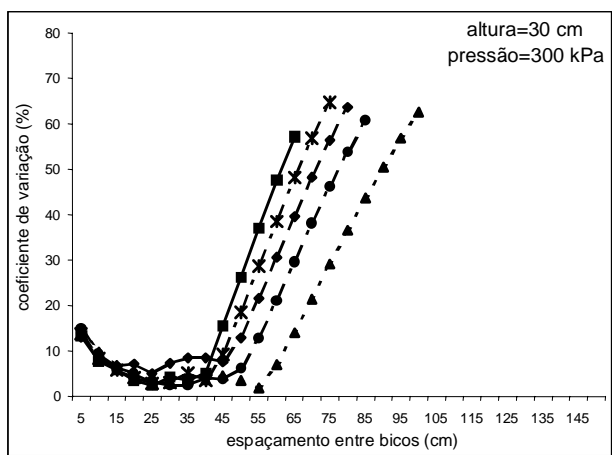

(B)

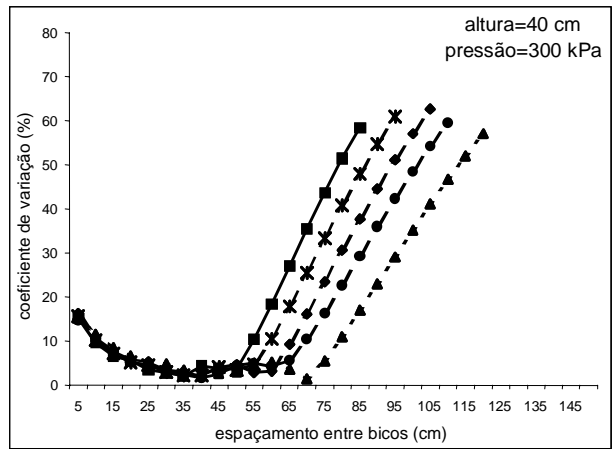

(D)

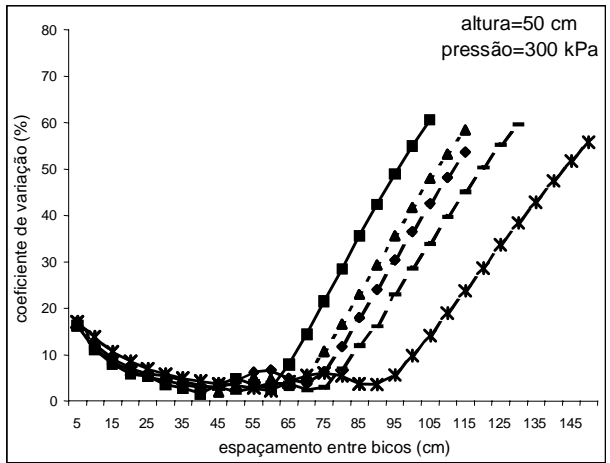

(E)

$$
\longrightarrow \text { Vertical } \quad \longrightarrow \rightarrow-30^{\circ} \text { horário } \quad \cdots-\cdots 30^{\circ} \text { anti-horário } \quad-\cdot *-45^{\circ} \text { horário } \quad--\bullet-\cdot 45^{\circ} \text { anti-horário }
$$

Figura 4 - Coeficiente de variação médio de pontas XR 8004 instaladas a 30 (A e B), 40 (C e D) e 50 cm de altura (E e F), a 200 (A, C e E) e $300 \mathrm{kPa}\left(\mathrm{B}, \mathrm{D}\right.$ e F), posicionadas a $30^{\circ}$ e $45^{\circ}$ nos sentidos horário e anti-horário e na vertical, em função do espaçamento entre bicos na barra pulverizadora. 


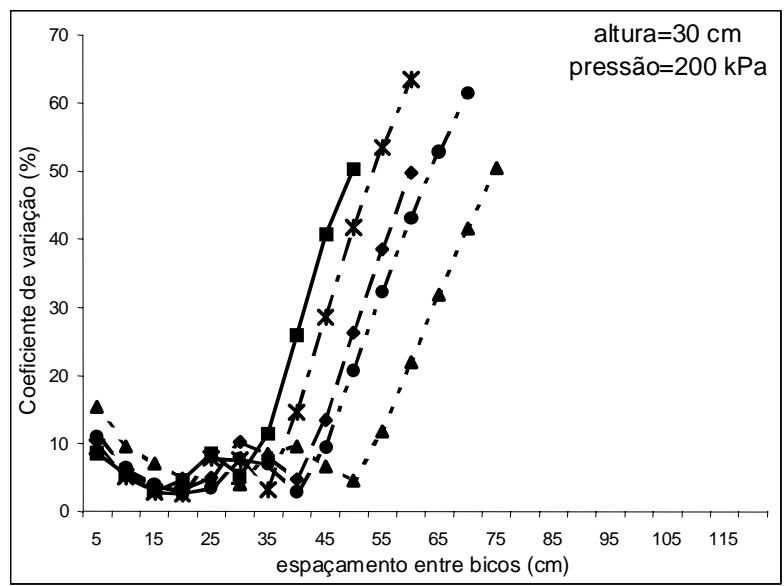

(A)

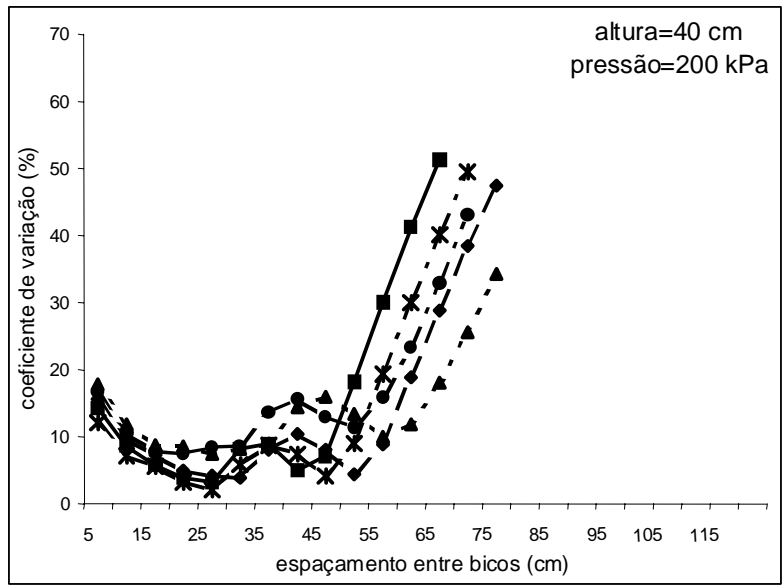

(C)

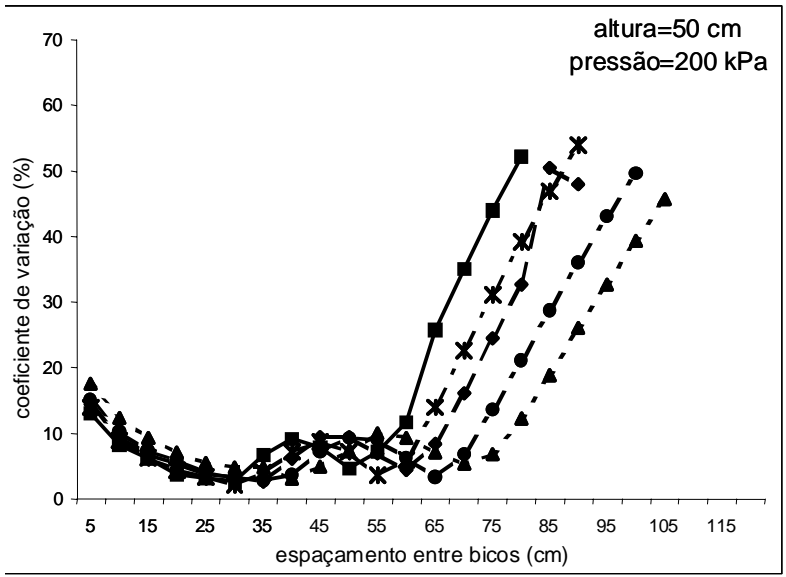

(E)

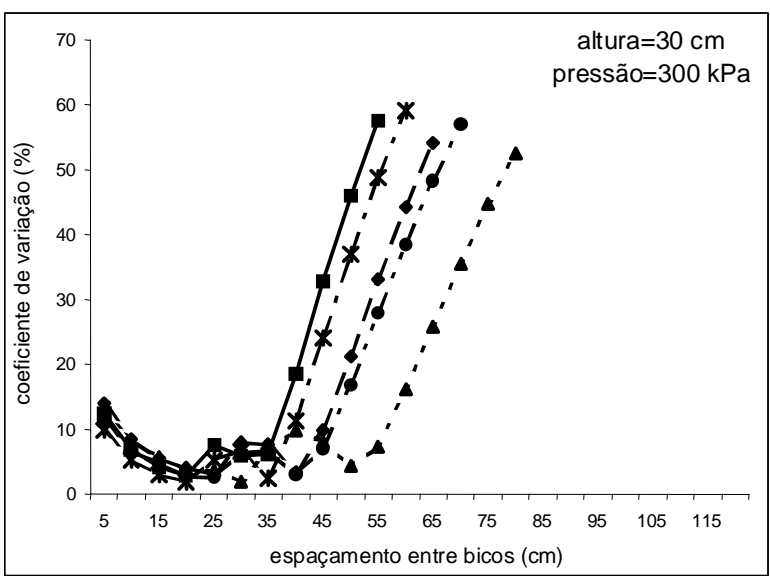

(B)

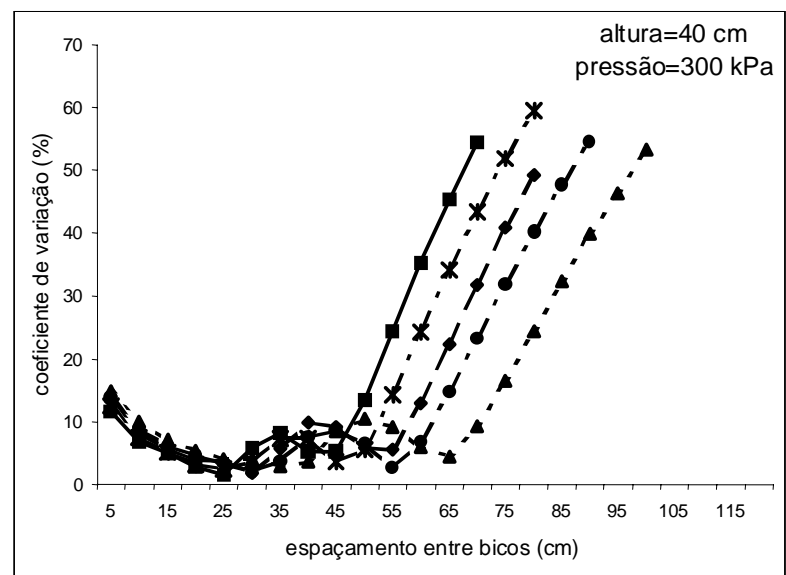

(D)

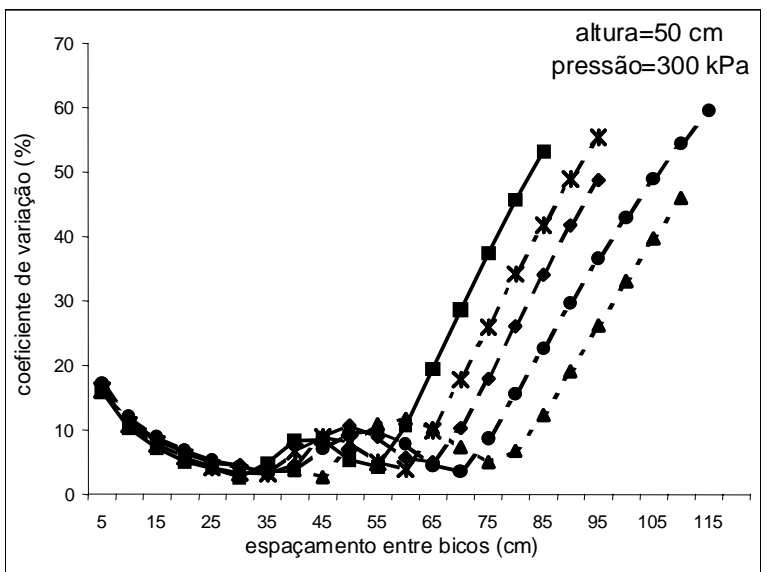

$(\mathrm{F})$

$\longrightarrow$ Vertical $\longrightarrow \rightarrow-30^{\circ}$ horário $-\rightarrow--45^{\circ}$ horário $-*--30^{\circ}$ anti-horário $-\rightarrow-45^{\circ}$ anti-horário

Figura 5 - Coeficiente de variação médio de pontas TP 8004 instaladas a 30 (A e B), 40 (C e D) e 50 cm de altura (E e F), a 200 (A, $\mathrm{C}$ e E) e $300 \mathrm{kPa}\left(\mathrm{B}, \mathrm{D}\right.$ e F), posicionadas a $30^{\circ}$ e $45^{\circ}$ nos sentidos horário e anti-horário e na vertical, em função do espaçamento entre bicos na barra pulverizadora. 

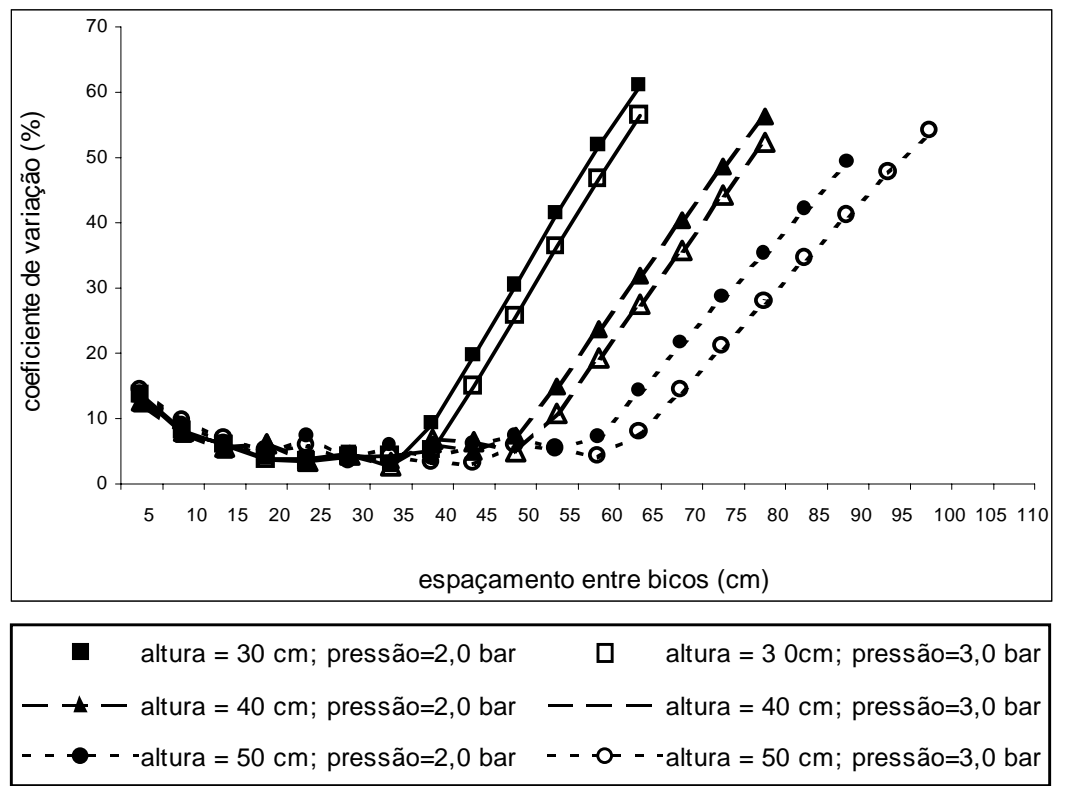

Figura 6 - Coeficiente de variação médio de pontas TJ60-8004 instaladas a 30 (A e B), 40 (C e D) e 50 cm de altura (E e F), a 200 (A, C e E) e 300 kPa (B, D e F), na posição vertical.

As Figuras 4, 5 e 6 reforçam essa constatação, pois, se o efeito da angulação do bico é o mesmo da elevação da barra, os resultados se mostraram similares também com relação ao CV. Esse fato foi demonstrado por Azimi et al. (1985) com pontas TeeJet 8003.

Os resultados aqui obtidos também indicam ser mais recomendáveis os espaçamentos menores, visto que há tendência de distribuição mais estável e uniformidade de distribuição menos influenciada por variações de altura e pressão.

Dessa forma, conclui-se que a angulação dos bicos na barra pulverizadora causa grande interferência no espaçamento entre bicos, independentemente da pressão, e que a uniformidade da distribuição da pulverização é menos influenciada pela altura da barra quando se usam menores espaçamentos entre bicos.

\section{LITERATURA CITADA}

AZIMI, A. H.; CARPENTER, T. G.; REICHARD, D. L. Nozzle spray distribution for pesticide application. Trans. Am. Soc. Agric. Eng., v. 28, p. 1410-1414, 1985.
BAUER, F. C. Distribuição e deposição da pulverização sob diferentes condições operacionais na cultura da soja (Glycine max (L.) Merrill). 2002. 130 f. Tese

(Doutorado em Agronomia/Proteção de Plantas) Universidade Estadual Paulista, Botucatu, 2002.

CHAPPLE, A. C.; HALL, F. R.; BISHOP, B. L. Assessment of single-nozzle patternation and extrapolation to moving booms. Crop Protection, v. 12, p. 207-213, 1993.

CHRISTOFOLETTI, J. C. Bicos de pulverização seleção e uso. Diadema: Spraying Systems do Brasil, 1997. $8 \mathrm{p}$.

DOWNER, R. A. et al. Herbicide spray distribution, quality and efficacy interactions: conflicts in requirements. Aspects Appl. Biol., n. 48, p. 79-89, 1997.

GALLI, J. C.; MATUO, T.; SIQUEIRA, E. C. Padrão de distribuição de alguns bicos hidráulicos. Planta Daninha, v. 4, n. 2, p. 144-150, 1983.

MATTHEWS, G. A. Pesticide application methods. 3.ed. London: Blackwell, 2000. 432 p.

PERECIN. D. et al. Padrões de distribuição obtidos com bicos Twinjet em função da altura e do espaçamento entre bicos. Eng. Agric., v. 14, p. 19-30, 1994.

PERECIN, D. et al. Padrões de distribuição obtidos com bicos TF-4, TJ60-11006 e TQ15006 em mesa de prova. Pesq. Agrop. Bras., v. 33, p. 175-182, 1998. 